\title{
Flavourful Inert Doublet Dark Matter
}

\section{Lopamudra Mukherjee ${ }^{\dagger *}$}

Department of Physics, Indian Institute of Technology Guwahati, Guwahati, Assam 781039, India.

E-mail: mukherjeelopa@iitg.ac.in

In this talk I discuss about some of the anomalous results in flavour physics and try to correlate them with the Inert Higgs doublet dark matter model. This is achieved by considering an extension of the Inert Higgs doublet model with $S U(2)_{L}$ singlet vector like fermions. Flavour data in $b \rightarrow s \ell \ell$ decay observables (like $R(K), R\left(K^{*}\right)$ ) and muon $(g-2)$ puts stringent bounds on the model parameters. The model can simultaneously satisfy relevant constraints in the dark matter sector and also abide by the bounds given by the ongoing direct detection experiments. I also discuss the discovery possibilities of such exotic particles in the future high luminosity (HL) runs of the LHC.

40th International Conference on High Energy Physics - ICHEP2020

July 28 - August 6, 2020

Prague, Czech Republic (virtual meeting)

\footnotetext{
${ }^{\dagger}$ I would like to thank the organisers of ICHEP 2020 for giving me the opportunity to present my work. I would also like to thank Basabendu Barman, Debasish Borah and Soumitra Nandi for fruitful collaboration.

*Speaker
} 


\section{Introduction}

One of the most intriguing mysteries of the Universe has been the presence of non-luminous baryonic matter in sufficiently large amounts (roughly five times that of ordinary matter) which is known as the dark matter (DM). Physicists have been trying to find a particle candidate for this dark matter even though any promising signature of its discovery has failed to show up in collider experiments. One of the most popular DM candidates belonging to the weakly interacting massive particle (WIMP) category, obtained from a simple extension of the Standard Model (SM) by an inert Higgs doublet $\left(\Phi_{2}\right)$, was first proposed by Ernest Ma [1]. Under an additional discrete $\mathbb{Z}_{2}$ symmetry, this scalar doublet transforms as $\Phi_{2} \rightarrow-\Phi_{2}$ while all other SM fields are even. Therefore, the lightest neutral odd scalar of this doublet can become a suitable DM candidate. For a detailed review on the model constraints and its phenomenology, please see [2]. However, even though the inert Higgs doublet model has been extensively studied from the point of view of DM, neutrino mass generation, collider physics, positron flux from cosmic ray signals etc, constraints from flavour physics has never been considered before.

In the flavour sector, there are reports of some anomalous results in the semileptonic flavour changing neutral current (FCNC) $b \rightarrow$ sll decays. In recent years, LHCb has measured the observables $[3,4]$

$$
R(K)=\frac{\mathcal{B}\left(B \rightarrow K \mu^{+} \mu^{-}\right)}{\mathcal{B}\left(B \rightarrow K e^{+} e^{-}\right)}=0.846_{-0.054-0.014}^{+0.060+0.016} \text {, for } q^{2} \in[1.1,6] \mathrm{GeV}^{2}
$$

and

$$
R\left(K^{*}\right)=\frac{\mathcal{B}\left(B \rightarrow K^{*} \mu^{+} \mu^{-}\right)}{\mathcal{B}\left(B \rightarrow K^{*} e^{+} e^{-}\right)}= \begin{cases}0.66_{-0.07}^{+0.11} \pm 0.03, & q^{2} \in[0.045,1.1] \mathrm{GeV}^{2}, \\ 0.69_{-0.07}^{+0.11} \pm 0.05, & q^{2} \in[1.1,6] \mathrm{GeV}^{2},\end{cases}
$$

where $q^{2}$ is the squared momentum of the leptons in the final state. Similar measurement of $R\left(K^{*}\right)$ by the Belle Collaboration is also there, but the data suffers from huge uncertainties. The above measurements deviate from their respective SM expectations by $\sim 2.5 \sigma$. In the SM, these processes occur at one-loop level in the SM and therefore deviations reported in their branching fraction measurements clearly hint at the presence of new physics (NP). This observed discrepancy calls for the need of some new mechanism that will generate lepton flavour universality violation (LFUV) either at tree level or via loops. NP contributions to the dimension six operators $O_{9}=$ $\frac{e^{2}}{g^{2}}\left(\bar{s} \gamma_{\mu} P_{L} b\right)\left(\bar{\ell} \gamma^{\mu} \ell\right)$ and $O_{10}=\frac{e^{2}}{g^{2}}\left(\bar{s} \gamma_{\mu} P_{L} b\right)\left(\bar{\ell} \gamma^{\mu} \gamma^{5} \ell\right)$ are known to be able to best explain the above discrepancy [5]. The branching fraction of the rare decay $B_{S} \rightarrow \mu^{+} \mu^{-}$with the same quark level transition, on the other hand, is known to be consistent with the SM prediction. Since the operator $O_{10}$ also contributes to this decay, $\mathcal{B}\left(B_{S} \rightarrow \mu^{+} \mu^{-}\right)$helps in putting severe constraints on the new physics parameter space. Hence, it is quite challenging to simultaneously accomodate both the SM consistent and inconsistent results in any NP model.

The anomalous magnetic moment of the muon is another important observable which calls for our attention. Even though both theory and experiment is known to a great accuracy, yet the difference between the two, given by [6]

$$
\Delta a_{\mu}=26.1(7.9) \times 10^{-10},
$$

signifies a $3.7 \sigma$ discrepancy. Studying a new physics model which is capable of addressing all the above results is therefore quite motivating. 


\section{Framework}

Since the inert Higgs doublet does not have tree level coupling with the SM fermions, it is not possible to address the above mentioned anomalous results with the inert Higgs alone. We therefore extend the inert Higgs dark matter model with three generations of down type vector-like quarks (VLQs) ' $D_{i}$ ', and singly charged vector-like leptons (VLLs) ' $E_{i}$ ' [7]. These additional fermions are also odd under a discrete $\mathbb{Z}_{2}$ symmetry which allows them to couple to SM fermions as

$$
\mathcal{L}_{Y} \supset\left(\lambda^{D}\right)_{i j} \bar{Q}_{i} \Phi_{2} D_{R j}+\left(\lambda^{E}\right)_{i j} \bar{L}_{i} \Phi_{2} E_{R j}+M_{D_{i}} \bar{D}_{L i} D_{R i}+M_{E_{i}} \bar{E}_{L i} E_{R i}+\text { h.c. },
$$

where $\lambda^{E, D}$ are the Yukawa couplings associated with the vector-like fermion (VLF) interactions and $(i, j)$ are the generation indices. In this setup, there will be contribution to $b \rightarrow s \ell \ell$ decays via box and penguin diagrams with a neutral inert scalar $\left(H^{0}, A^{0}\right)$ and VLFs in the loop which modifies the Wilson coefficients $C_{9}$ and $C_{10}$ corresponding to the effective operators mentioned previously. As one can see from Eq. 4 , the new couplings $\left(\lambda_{i j}^{E, D}\right)$ are generation dependent and therefore depending on the type of VLF in the loop, there will be several contributions to the decay amplitude. For the simplicity of the analysis, we have assumed the hierarchy: $\lambda_{i j}^{E, D}<<\lambda_{i i}^{E, D}$ (i, $\mathrm{j}$ $=1,2$ and 3), i.e, the off diagonal Yukawas are suppressed with respect to the diagonal terms. Also, since we require lepton universality violation, we have further assumed $\lambda_{33}^{E, D}>>\lambda_{22}^{E, D}>>\lambda_{11}^{E, D}$. Under this simplified scenario, the box diagram with $D_{3}$ and $E_{2}$ (in the loop) will have the dominant contribution to the process $b \rightarrow s \mu^{+} \mu^{-}$. To simplify our notations, we will rewrite our relevant couplings as : $\lambda_{33}^{D} \equiv \lambda_{b}, \lambda_{23}^{D} \equiv \lambda_{s}, \lambda_{13}^{D} \equiv \lambda_{d}, \lambda_{11}^{E} \equiv \lambda_{e}, \lambda_{22}^{E} \equiv \lambda_{\mu}$ and $\lambda_{33}^{E} \equiv \lambda_{\tau}$.

From the Lagrangian in Eq. 4, it is evident that there will be contributions to lepton flavour violating (LFV) decays $\ell_{i} \rightarrow \ell_{j} \gamma$ as well as $\Delta a_{\mu}$ through vertex correction diagrams involving the inert scalars and VLLs. Once again, due to our choice of hierarchy in the couplings, the dominant contribution to the muon magnetic moment will come from $E_{2}$ in the loop. We have also considered the $B_{s}-\bar{B}_{s}$ mixing since some of our model parameters are sensitive to this $\Delta F=2$ process. In our model the dominant contribution to the mass difference $\Delta M_{B_{s}}$ will come from the box diagram. The current data on $\Delta M_{B_{s}}$ allows NP as large as (15-20)\%.

As mentioned before, the lightest odd neutral scalar of the inert doublet will be the DM candidate, which we consider to be $H^{0}$. Therefore all the relevant observables mentioned above will be sensitive to the mass of the DM. However, due to the addition of the VLFs, there are now additional annihilation channels that will contribute to the DM relic abundance and DM-nucleon scattering cross-section. For more details, refer [7].

\section{Results}

With the simple extention of the inert doublet model (IDM) mentioned above, we study the combined parameter space which satisfies not only the DM relic and direct detection (DD) constraints, but also explains the flavour data. We know that the inert doublet DM satisfies the relic only in two specific mass ranges of the DM; one when $M_{H^{0}}$ is low (between $50-90 \mathrm{GeV}$ ) and the other when $M_{H^{0}}>500 \mathrm{GeV}$ [2]. Even though the dark sector is now modified, the intermediate mass region of the inert doublet remains barren even with the addition of the VLFs. Therefore we focus on the low and high mass regions only. 


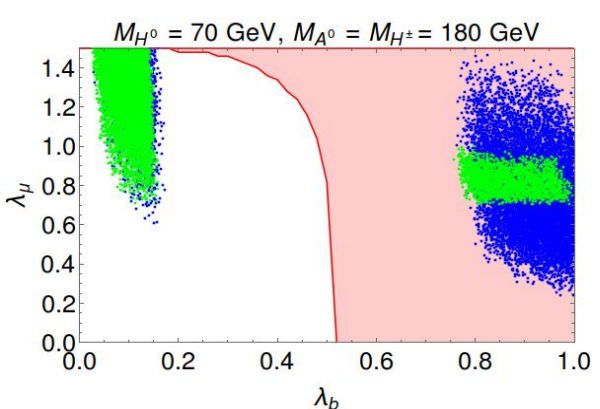

(a)

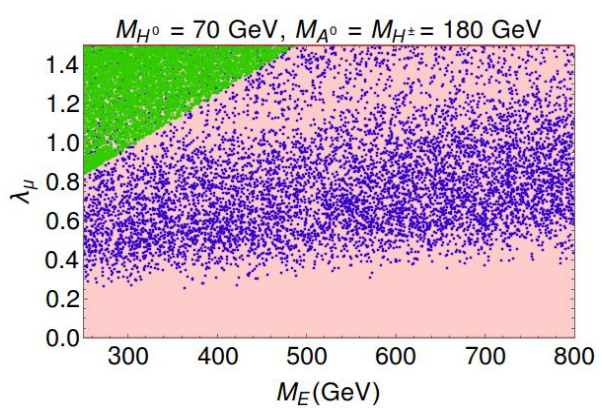

(c)

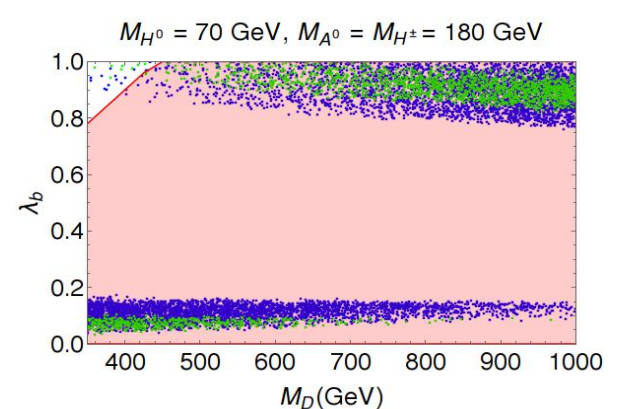

(b)

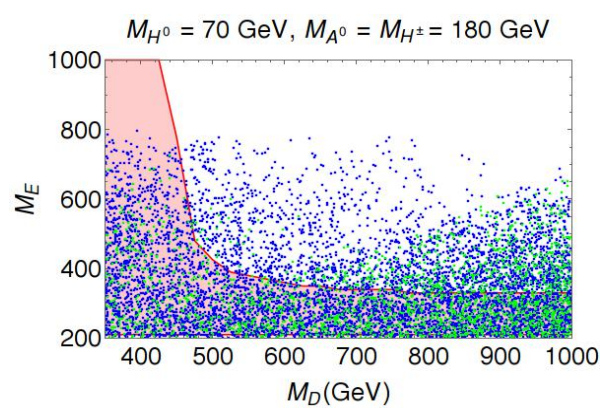

(d)

Figure 1: Correlation between different NP parameters for a low mass DM at $M_{H^{0}}=70 \mathrm{GeV}$. The blue points satisfy all relevant flavour constraints within their $2 \sigma$ confidence intervals except muon (g-2) anomaly. When we further apply the $\Delta a_{\mu}$ bounds then the allowed region shrinks to the green points. The red regions satisfy the relic and direct search constraints when we consider degenerate vector-like fermion masses.

In order to simplify the analysis, we have fixed few of the couplings, such as $\lambda_{s}=0.01$ and $\lambda_{e}=0.001$ and choose $\lambda_{b}<1, \lambda_{\mu}<1.5$ and $\lambda_{\tau} \approx 1.5$. Also, without any loss of generality, we assume that the VLQs and VLLs are mass degenerate. The rest of the free parameters are constrained from the $R\left(K^{(*)}\right), \mathcal{B}\left(B_{s} \rightarrow \mu \mu\right), \Delta a_{\mu}$ and relic data. With these choices of the couplings, we can easily overcome the present constraints from $B_{s}-\bar{B}_{s}$ mixing, $\mathcal{B}\left(B \rightarrow X_{s} \gamma\right)$ etc. Also, as mentioned before, we choose the off diagonal couplings to be much suppressed compared to the diagonal ones. This allows us to easily abide by the bounds on LFV decays such as $\tau \rightarrow \mu \gamma, \mu \rightarrow e \gamma$ etc. In pure IDM, the electroweak precision observables (EWPO) like $S$ and $T$ constrain the mass splitting $\Delta M$ between the inert scalars [8] which is taken into account. The singlet vector fermions in our model do not mix and therefore there will not be any additional significant contribution in $S, T$ and $U$ parameters.

We present our main results for the low mass DM in Fig. 1. As expected, the major constraints on the new parameters are mainly coming from the flavour data, in particular from $\mathcal{B}\left(B_{s} \rightarrow \mu \mu\right)$. The blue scattered points satisfy the data on $R(K), R\left(K^{*}\right)$ and $\mathcal{B}\left(B_{s} \rightarrow \mu \mu\right)$ in their respective $2 \sigma$ confidence intervals which shrink to the green points when we also consider $\Delta a_{\mu}$ as a constraint. Here, we have considered the excess in $\Delta a_{\mu}$ within its 3- $\sigma$ range. The red regions represent the parameter space satisfying the relic density and direct detection cross section bounds. The interesting feature here is the presence of two distinct regions in the parameter spaces of $\lambda_{\mu}, \lambda_{b}$ and 
$M_{D}$ (Fig. 1(a) and 1(b)). These two regions correspond to high and low values of $\lambda_{b}$, respectively. In both the allowed regions, $\lambda_{\mu}$ can take moderate values $>0.5$. However, its magnitude can not be very high ( $>>1$ ) when $\lambda_{b} \gtrsim 0.7$. On the other hand when $\lambda_{b}$ is small, the common parameter spaces are obtained in regions where $\lambda_{\mu} \approx 1.5$, which is seen to be relaxed if we lift the mass degeneracies of the VLLs.

We perform a similar analysis for the high mass DM and interestingly we find that, in this case, we will be able to explain the $R\left(K^{(*)}\right)$ anomaly only for higher values of $\lambda_{\mu}(\approx 3$ or 4$)$. However, for the same masses, such high values of $\lambda_{\mu}$ makes the relic under-abundant. Hence, it is not possible to obtain a common parameter space that satisfies both relic abundance and flavour constraints simultaneously. So we discard further investigations for this case.

\section{Collider Searches}

Due to the presence of the exotic VLFs, the model gives rise to several exciting collider signatures. Among these, the most important ones are the dilepton plus missing energy $\left(E_{T}\right)$ and dijet plus $E_{T}$ (with and without b-jet tagging). From the parameter space obtained in Fig. 1, we choose some suitable benchmark points and study their signal prospects for the high luminosity (HL) runs of the Large Hadron Collider (LHC). For this, we first implemented the model in FeynRule [9]. After generating the parton level events at $\sqrt{s}=14 \mathrm{TeV}$ in MADGRAPH [10], we further shower them through PYTHIA [11] for hadronization. Appropriate $K$-factors have been used to match them with the Next-to-Leading order (NLO) cross section. The dominant SM backgrounds for the above signals are $t \bar{t}, W^{+} W^{-}, W^{ \pm} Z, Z Z, W j, Z j$ and Drell - Yan. Some basic cuts and reconstruction criteria have been applied in order to simulate the environment of the LHC. The details can be found in [7]. We have also made a comparative study with the collider aspects of the pure IDM model in order to discriminate our model from it.

We have used $M_{\ell \ell}, E_{T}$ and the scalar sum of all isolated lepton/jet transverse momentum $\left(H_{T}\right)$ as observables in order to distinguish the signals from the SM backgrounds. For the $\left(\ell^{+} \ell^{-}+E_{T}\right)$ final state, we observe a long tail in the $M_{\ell \ell}$ and $H_{T}$ distributions of the signals compared to a flat SM background or even a pure IDM signal. This is because the source of the leptons in the final state are the heavy new particles apart from the SM gauge bosons and so they are much more boosted than those coming only from the SM gauge bosons. From our analysis, we find a lower bound on the VLF masses. VLQ below $500 \mathrm{GeV}$ and VLL below $250 \mathrm{GeV}$ are found to be ruled out from the current LHC data. Some of the benchmarks also have discovery potential in the future HL-LHC. Although the non-observance of any excess in the data in future will help us to rule out higher mass regions of the vector like fermions, $M_{D} \gtrsim 900 \mathrm{GeV}$ will be beyond the reach of even the proposed HL-LHC. This makes the model difficult to be probed in $2 j+E_{T}$ final state even at high luminosity LHC runs.

\section{Summary}

We have extended the inert Higgs dark matter model with suitable VLFs in order to explain present data in flavour physics through its unique connection with the dark sector. Apart from satisfying different flavour constraints, we also make some new predictions for the VLF masses and 
couplings. Although we have mostly focussed on the neutral current decay modes of the $B$-meson, the model is also capable of addressing the data in the flavour changing charged current decays such as $b \rightarrow c \ell \bar{v}_{\ell}$, the results of which will be published soon [12]. This will put further stringent constraints on the model parameters and may be useful to rule out a part of the parameter space. The search for exotic new particles can then become more focussed.

\section{References}

[1] N. G. Deshpande and E. Ma, "Pattern of Symmetry Breaking with Two Higgs Doublets", Phys. Rev. D 18 (1978), 2574 doi:10.1103/PhysRevD.18.2574

[2] M. Gustafsson, "The Inert Doublet Model and Its Phenomenology", PoS CHARGED2010 (2010), 030 doi:10.22323/1.114.0030 [arXiv:1106.1719 [hep-ph]].

[3] R. Aaij et al. [LHCb], "Search for lepton-universality violation in $B^{+} \rightarrow K^{+} \ell^{+} \ell^{-} d e$ cays", Phys. Rev. Lett. 122 (2019) no.19, 191801 doi:10.1103/PhysRevLett.122.191801 [arXiv:1903.09252 [hep-ex]];

[4] R. Aaij et al. [LHCb], "Test of lepton universality with $B^{0} \rightarrow K^{* 0} \ell^{+} \ell^{-}$decays", JHEP $\mathbf{0 8}$ (2017), 055 doi:10.1007/JHEP08(2017)055 [arXiv:1705.05802 [hep-ex]].

[5] S. Bhattacharya, A. Biswas, S. Nandi and S. K. Patra, Phys. Rev. D 101 (2020) no.5, 055025 doi:10.1103/PhysRevD.101.055025 [arXiv:1908.04835 [hep-ph]].

[6] P.A. Zyla et al. [Particle Data Group], PTEP 2020 (2020) no.8, 083C01 doi:10.1093/ptep/ptaa104

[7] B. Barman, D. Borah, L. Mukherjee and S. Nandi, "Correlating the anomalous results in $b \rightarrow s$ decays with inert Higgs doublet dark matter and muon $(g-2)$ ", Phys. Rev. D 100 (2019) no.11, 115010 doi:10.1103/PhysRevD.100.115010 [arXiv:1808.06639 [hep-ph]].

[8] R. Barbieri, L. J. Hall and V. S. Rychkov, "Improved naturalness with a heavy Higgs: An Alternative road to LHC physics", Phys. Rev. D 74, 015007 (2006) doi:10.1103/PhysRevD.74.015007 [hep-ph/0603188].

[9] A. Alloul, N. D. Christensen, C. Degrande, C. Duhr and B. Fuks, Comput. Phys. Commun. 185, 2250 (2014) doi:10.1016/j.cpc.2014.04.012 [arXiv:1310.1921 [hep-ph]];

[10] J. Alwall et al., JHEP 1407 (2014) 079 doi:10.1007/JHEP07(2014)079 [arXiv:1405.0301 [hep-ph]];

[11] T. Sjostrand, S. Mrenna and P. Z. Skands, JHEP 0605, 026 (2006) doi:10.1088/11266708/2006/05/026 [hep-ph/0603175].

[12] B. Barman, D. Borah, L. Mukherjee and S. Nandi, in preparation. 